\title{
Is There Evidence for EEG-Neurofeedback Specificity in the Treatment of Internalizing Disorders? A Protocol for a Systematic Review and Meta-Analysis
}

\author{
Tyson Michael Perez ${ }^{*}$, Paul Glue ${ }^{2}$, Divya B Adhia ${ }^{3}$, Jerin Mathew ${ }^{4}$, and Dirk De Ridder 5 \\ ${ }^{1}$ Departments of Surgical Sciences \& Psychological Medicine, University of Otago, Dunedin, New Zealand \\ ${ }^{2}$ Department of Psychological Medicine, University of Otago, Dunedin, New Zealand \\ ${ }^{3}$ Department of Surgical Sciences, University of Otago, Dunedin, New Zealand \\ ${ }^{4}$ School of Physiotherapy, University of Otago, Dunedin, New Zealand \\ ${ }^{5}$ Department of Surgical Sciences, University of Otago, Dunedin, New Zealand
}

\begin{abstract}
Background: Mental illnesses are increasing worldwide with the internalizing disorders (IDs; e.g., anxiety disorders, depressive disorders) being the most prevalent. Current first-line therapies (e.g., pharmacotherapy) offer high failure rates and substantial side effects. Electroencephalographic neurofeedback (EEG-NFB) has been shown to be an effective and safe treatment for these conditions; however, there remains much doubt regarding the existence of specificity (i.e., clinical effects specific to the modulation of the EEG variables of interest). This is a protocol for a quantitative review that will attempt to determine if there is evidence for EEGNFB specificity in the treatment of IDs. Methods: We will consider all published and unpublished randomized, double-blind (i.e., trainees and raters), sham/placebo-controlled (i.e., feedback contingent on a random signal, the activity from a different person's brain, or an unrelated signal from the trainee's own brain) trials involving humans with at least one ID diagnosis without exclusion by language, locality, ethnicity, age, or sex. Effect sizes will be calculated for individual studies and combined in a meta-analysis. Discussion: This protocol outlines the research methodology for a quantitative review undertaken to assess for evidence of EEG-NFB specificity in the treatment of IDs. Registration: This review was registered with the International Prospective Register of Systematic Reviews (PROSPERO; registration number: CRD42020159702).
\end{abstract}

Keywords: EEG; neurofeedback; internalizing disorders; emotional disorders; affective disorders

Citation: Perez, T. M., Glue, P., Adhia, D. B., Mathew, J., De Ridder, D. (2021). Is there evidence for EEG-neurofeedback specificity in the treatment of internalizing disorders? A protocol for a systematic review and meta-analysis. NeuroRegulation, 8(1), 22-28. https://doi.org/10.15540/nr.8.1.22

*Address correspondence to: Tyson M Perez, Section of Neurosurgery, Department of Surgical Sciences, Dunedin School of Medicine, Dunedin Hospital, 201 Great King Street, Dunedin 9016, New Zealand. Email: tyson.perez@postgrad.otago.ac.nz

Copyright: ( 2021. Perez et al. This is an Open Access article distributed under the terms of the Creative Commons Attribution License (CC-BY).
Edited by:

Rex L. Cannon, PhD, SPESA Research Institute, Knoxville, Tennessee, USA

Reviewed by:

Randall Lyle, PhD, Mount Mercy University, Cedar Rapids, lowa, USA

Estate M. Sokhadze, PhD, University of South Carolina, School of Medicine Greenville, Greenville, South Carolina, USA

\section{Background and Rationale}

Internalizing disorders (IDs; e.g., anxiety disorders, ANX; depressive disorders, DEP; posttraumatic stress disorder, PTSD; obsessive-compulsive disorder, OCD) are the most prevalent psychopathologies (Demyttenaere et al., 2004; Kessler et al., 2009; Kessler et al., 2007; Kessler et al., 2005; Wells et al., 2006) and can be broadly characterized by a proclivity to direct distress inwardly (Buchan, Sunderland, Carragher, Batterham, \& Slade, 2014; Carragher, Krueger, Eaton, \& Slade, 2015; Kotov et al., 2017; Krueger \& Eaton, 2015; Rhee, Lahey, \& Waldman, 2015). There are numerous shortcomings with traditional frontline ID treatments (i.e., pharmacotherapy and psychotherapy) including substantial long-term failure rates (Haller, Cohen Kadosh, Scerif, \& Lau, 2015; James, James, Cowdrey, Soler, \& Choke, 2015; Peters, Dunlop, \& Downar, 2016; Pinter et al., 
2019), lack of access (Andrade et al., 2014; Bandelow \& Michaelis, 2015; Haller et al., 2015; Möller et al., 2016; Schoenberg \& David, 2014), and marked adverse side effects (Alvares, Quintana, Hickie, \& Guastella, 2016; Haller et al., 2015; Möller et al., 2016; Pinter et al., 2019; Tiller, 2013). Moreover, a decades-long drought in the discovery of new agents has prompted pharmaceutical companies to abandon the neuropsychiatric space (Buzsáki \& Watson, 2012), leading to appeals from around the world for innovative interventions (Haller et al., 2015; Kris, 2018; Lancet Global Mental Health Group et al., 2007; Pinter et al., 2019).

With aberrations in the brain's electrical activity well recognized in IDs (Alhaj, Wisniewski, \& McAllisterWilliams, 2010; Buzsáki \& Watson, 2012; losifescu, 2011; Jokić-Begić \& Begić, 2003; Pizzagalli et al., 2002; Wahbeh \& Oken, 2013), electroencephalographic neurofeedback (EEG-NFB) has been touted as a possible solution. EEG-NFB is a noninvasive form of biofeedback that teaches the brain to modify its function via a closed-loop braincomputer interface, whereby an exogenous sensory stimulus (e.g., audible tone) is fed back to the participant in real time following some predetermined electrical activity recorded from the scalp (Arns et al., 2017; Collura, 2013; Marzbani, Marateb, \& Mansourian, 2016; Orndorff-Plunkett, Singh, Aragón, \& Pineda, 2017; Sitaram et al., 2016). EEG-NFB is widely believed to work predominantly through operant conditioning, a type of associative learning whereby the probability of some given electrical behavior is modified via a temporally associated reinforcing stimulus (Alkoby, Abu-Rmileh, Shriki, \& Todder, 2018; EnriquezGeppert, Huster, \& Herrmann, 2017; OrndorffPlunkett et al., 2017). Although the use of EEG-NFB for IDs in routine clinical psychiatric practice has yet to receive widespread support (Arns et al., 2017; Begemann, Florisse, van Lutterveld, Kooyman, \& Sommer, 2016; Omejc, Rojc, Battaglini, \& Marusic, 2019), there is substantial evidence that EEG-NFB is efficacious (e.g., Askovic et al., 2019; Bell, Moss, \& Kallmeyer, 2019; Cheon et al., 2017; Chiba et al., 2019; Hou et al., 2021; Noohi, Miraghaie, Arabi, \& Nooripour, 2017; Orndorff-Plunkett et al., 2017; Panisch \& Hai, 2018; Reiter, Andersen, \& Carlsson, 2016; Ros et al., 2017; Schoenberg \& David, 2014; Tolin, Davies, Moskow, \& Hofmann, 2020; van der Kolk et al., 2016; Wang et al., 2019).

That said, skeptics claim that EEG-NFB's effects stem entirely from nonspecific factors (e.g., expectations, demand characteristics, context) based on multiple randomized, sham/placebo- controlled trials of attention-deficit/hyperactivity disorder (ADHD) showing comparable clinical improvements in both experimental and control groups (Ghaziri \& Thibault, 2019; Neurofeedback Collaborative Group et al., 2020; Schönenberg et al., 2017a, 2017b; Thibault, Lifshitz, \& Raz, 2016; Thibault, Veissière, Olson, \& Raz, 2018). Among other criticisms, EEG-NFB proponents point out that evidence of EEG-learning (i.e., improvement in the targeted electrophysiological variable) in the active groups and a lack thereof in the controls, considered by many a prerequisite for the evaluation EEGNFB's specificity (Arns, Heinrich, \& Strehl, 2014; Holtmann, Sonuga-Barke, Cortese, \& Brandeis, 2014; Kerson \& Collaborative Neurofeedback Group, 2013; Sherlin et al., 2011; Szewczyk, Ratomska, \& Jaśkiewicz, 2018; Witte, Kober, \& Wood, 2018; Zuberer, Brandeis, \& Drechsler, 2015), was conspicuously absent in the trials presented as evidence for wholly nonspecific effects (Pigott, Cannon, \& Trullinger, 2018; Trullinger, Novian, Russell-Chapin, \& Pradhan, 2019).

\section{Objectives}

The aim of our review is to comprehensively evaluate all relevant and available ID-focused randomized, double-blind, sham/placebo-controlled trials for evidence of EEG-NFB specificity via clinical outcome measures.

\section{Eligibility Criteria}

We will consider all EEG-NFB published and unpublished trials involving humans with at least one ID diagnosis per the Diagnostic and Statistical Manual of Mental Disorders (DSM; American Psychiatric Association, 2013) or the International Classification of Diseases (ICD; World Health Organization, 2018) with no exclusion by language, locality, ethnicity, age, or sex. To minimize bias and control for nonspecific effects, all trials must be randomized, double-blind (trainees and raters), and sham/placebo-controlled (i.e., feedback contingent on a random signal, the activity from a different person's brain, or an unrelated signal from the trainee's own brain).

\section{Information Sources}

Studies eligible for review will be identified in a literature search from earliest dates within multiple databases including Scopus, PubMed, Ovid MEDLINE, Cochrane Central Register of Controlled Trials (CENTRAL), Embase, Allied and Complementary Medicine (AMED), Psyclnfo, and PsycExtra. The electronic database searches will be supplemented by searching for trial protocols 
through the World Health Organization's International Clinical Trials Registry Platform (ICTRP), ClinicalTrials.gov, and the Australia New Zealand Clinical Trials Registry (ANZCTR). Additionally, citation lists of relevant articles and previous systematic reviews will be hand-searched for trials meeting our criteria but not located by the electronic database searches.

\section{Search Strategy}

The search strategies were peer reviewed by the University's Health Sciences Librarian with expertise in systematic review searching but not otherwise associated with the project. Literature search strategies were developed using medical subject heading (MeSH) and text words related to internalizing disorders and neurofeedback. When possible, limits imposed included participant type (i.e., human) and study design (e.g., randomized controlled trial, controlled clinical trial). As an example, our search strategy for Ovid MEDLINE will use exploded subject headings linked by Boolean operators (i.e., OR, AND) as follows: $\exp$ depression/ OR exp anxiety/ OR exp fear/ OR exp anxiety disorders/ OR exp mood disorders/ OR exp neurotic disorders/ OR exp "Trauma and Stressor Related Disorders" OR exp anorexia/ OR "Feeding and Eating Disorders"/ AND exp Biofeedback, Psychology/ with limits Humans and Randomized Controlled Trial. A detailed account of the search strategies for the various databases can be found in Supplement 1.

Data Management, Selection Process, and Data Collection Process

A single reviewer will collate the list of possible studies for inclusion and export them to EndNote (version X9) where duplicates will be removed. Two independent reviewers (TP \& JM) will screen titles and abstracts for eligibility. Each reviewer will independently assess full reports of trials that appear to meet the inclusion criteria, or where there is any uncertainty. We will seek additional information from study authors, via a maximum of three email requests, where necessary to resolve questions regarding eligibility. Disagreements will be resolved in discussion between TP and JM, otherwise a third team member (DA) will become involved to make the final decision. Reasons for excluding trials will be recorded. The independent reviewers will not be blinded to the journal titles, trial authors, or institutions. Data will be extracted by independent reviewers (TP \& JM) via a table generated in Word (Microsoft 365). A synthesis of the findings will be generated.

Data Items
The data items extracted will include (a) first author and publication/completion year, (b) primary condition(s) under study, (c) participant demographics (e.g., ages, sexes, etc.), (d) sham/placebo type, (e) EEG-NFB protocol (e.g., targets, reward rate, number/frequency/duration of sessions), (f) clinical outcome measure, and (g) evidence of targeted EEG-learning.

\section{Outcomes and Prioritization}

Our primary outcome of interest is between-group mean difference in change/final scores collected from clinician ratings or self/parent/teacher reports. In the event of a combination of the latter, the order of preference is self $>$ parent $>$ teacher. In the case of multiple rating scales for a given condition, the scale querying the most central aspects of the condition under study will be selected. In the case of multiple values for a single scale (i.e., total vs. subscale scores), total scores will be used. In the case of multiple posttreatment data collection time points, values obtained furthest from treatment termination will be given preference as it is believed that long-term outcomes may help to clarify the issue of specificity (Van Doren et al., 2019). To date, standard EEG-NFB protocols have not been established for the treatment of IDs (Banerjee \& Argáez, 2017); therefore, no protocols will be excluded.

\section{Risk of Bias in Individual Studies}

Two independent reviewers (TMP \& JM) will assess the risk of bias using the Cochrane Risk of Bias tool version 2 (RoB 2) which covers five domains (domain 1: risk of bias arising from the randomization process; domain 2: risk of bias due to deviations from the intended interventions; domain 3: risk of bias due to missing outcome data; domain 4: risk of bias in measurement of the outcome; domain 5: risk of bias in the selection of the reported result) as well an overall risk of bias. A judgment as to the possible risk of bias (i.e., low, some concerns, or high) on each of the domains will be made from the report. If there is insufficient detail reported in the study, the original study investigators will be contacted for more information. These judgements will be made based on the criteria for judging the risk of bias (Higgins et al., 2020). Disagreements will be resolved in discussion between TMP and JM, otherwise a third team member (DA) will become involved to make the final decision.

\section{Synthesis}

If enough studies are available, a meta-analysis will be performed utilizing inverse variance and random effects modelling to generate an overall 
standardized mean difference $(95 \% \mathrm{Cl})$. Effect sizes $(95 \% \mathrm{Cl})$ will be calculated and displayed in a forest plot using RevMan (version 5.4.1). In cases of missing data, we will attempt to contact the trial authors to obtain the missing data. Statistical heterogeneity will be tested using the $\mathrm{Chi}^{2}$ test (significance level: 0.1 ) and $\mathrm{I}^{2}$ statistic (0\% to $40 \%$ : might not be important; 30\% to $60 \%$ : may represent moderate heterogeneity; $50 \%$ to $90 \%$ : may represent substantial heterogeneity; $75 \%$ to $100 \%$ : considerable heterogeneity). If high levels of heterogeneity among the trials exist $\left(I^{2}>=50 \%\right.$ or $p$ $<0.1$ ), important characteristics of the included studies (e.g., overall level of bias) will be analyzed via meta-regression or sensitivity analysis to try to explain the source of heterogeneity.

\section{Meta-bias(es)}

The potential for publication and small sample biases will be explored by funnel plots and Egger's test if $\geq 10$ studies are available.

\section{Confidence in Cumulative Evidence}

The quality of the cumulative evidence will be assessed using the Grading of Recommendations, Assessment, Development and Evaluations (GRADE). Quality will be adjudicated as high (there is a lot of confidence that the true effect lies close to that of the estimated effect), moderate (the true effect is probably close to the estimated effect), low (the true effect might be markedly different from the estimated effect), or very low (the true effect is likely to be substantially different from the estimated effect).

\section{Discussion}

Neuropsychiatric disorders are among the most common causes of morbidity and mortality (Kessler et al., 2009) with rates markedly increasing worldwide in recent years (Duffy, Twenge, \& Joiner, 2019; Haidt \& Allen, 2020; Keyes, Gary, O'Malley, Hamilton, \& Schulenberg, 2019; Pfeifer \& Allen, 2020; Twenge, Cooper, Joiner, Duffy, \& Binau, 2019). Among them, the IDs, which are characterized by distress experienced inwardly (Buchan et al., 2014; Cosgrove et al., 2011), are the most prevalent. Recently, a government inquiry here in New Zealand has shed light on the shortcomings of traditional frontline treatments (e.g., pharmacotherapy) and called for wider implementation of nonpharmaceutical approaches in treatment of mental health problems (Kris, 2018). Moreover, scientists around the world are calling for research into "novel interventions that may be based on altering plasticity or returning circuitry rather than neurotransmitter pharmacology" (Insel \& Wang, 2010). EEG-NFB appears to be a safe, noninvasive, and efficacious that can be used as an adjunct or stand-alone treatment; however, there are questions regarding the nature of those effects. Specifically, there is much controversy surrounding the existence of specific effects. We hope that our review helps bring some clarity to this debate.

\section{Author Declarations}

This systematic review is part of a $\mathrm{PhD}$ thesis supported by the Department of Surgical Sciences, University of Otago, Dunedin, New Zealand. The department had no role in the design, implementation, analyses, interpretation, or dissemination of the results. All data generated or analyzed during this study are included in this published article and its supplementary information files. The authors declare that they have no competing interests. TMP is the guarantor and drafter of the manuscript. All authors contributed to the development of the selection criteria, the risk of bias assessment strategy and data extraction criteria. TMP developed and implemented the search strategy. PG provided expertise on mental health disorders. JM assisted with the article selections. All authors read, provided feedback, and approved the final manuscript.

\section{Author Acknowledgements}

We would like to thank the University of Otago Health Sciences librarian, Christy Ballard, for her help in designing the search strategy.

\section{References}

Alhaj, H., Wisniewski, G., \& McAllister-Williams, R. H. (2010). The use of the EEG in measuring therapeutic drug action: Focus on depression and antidepressants. Journal of Psychopharmacology, 25(9), 1175-1191. https://doi.org $/ 10.1177 / 0269881110388323$

Alkoby, O., Abu-Rmileh, A., Shriki, O., \& Todder, D. (2018). Can we predict who will respond to neurofeedback? A review of the inefficacy problem and existing predictors for successful EEG neurofeedback learning. Neuroscience, 378, 155-164. https://doi.org/10.1016/j.neuroscience.2016.12.050

Alvares, G. A., Quintana, D. S., Hickie, I. B., \& Guastella, A. J. (2016). Autonomic nervous system dysfunction in psychiatric disorders and the impact of psychotropic medications: A systematic review and meta-analysis. Journal of Psychiatry \& Neuroscience, 41(2), 89-104. https://doi.org/10.1503 /jpn.140217

American Psychiatric Association. (2013). Diagnostic and statistical manual of mental disorders (5th ed.). Washington, DC: Author.

Andrade, L. H., Alonso, J., Mneimneh, Z., Wells, J. E., AlHamzawi, A., Borges, G., ... Kessler, R. C. (2014). Barriers to mental health treatment: Results from the WHO World Mental Health surveys. Psychological Medicine, 44(6), 1303-1317. https://doi.org/10.1017/S0033291713001943 
Arns, M., Batail, J.-M., Bioulac, S., Congedo, M., Daudet, C., Drapier, D., ... The NExT group. (2017). Neurofeedback: One of today's techniques in psychiatry? L'Encéphale, 43(2), 135145. https://doi.org/10.1016/j.encep.2016.11.003

Arns, M., Heinrich, H., \& Strehl, U. (2014). Evaluation of neurofeedback in ADHD: The long and winding road. Biological Psychology, 95, 108-115. https://doi.org/10.1016 /j.biopsycho.2013.11.013

Askovic, M., Watters, A. J., Coello, M., Aroche, J., Harris, A. W. F., \& Kropotov, J. (2019). Evaluation of neurofeedback for posttraumatic stress disorder related to refugee experiences using self-report and cognitive ERP measures. Clinical EEG and Neuroscience, 51(2), 79-86. https://doi.org/10.1177 $/ 1550059419849170$

Bandelow, B., \& Michaelis, S. (2015). Epidemiology of anxiety disorders in the 21st century. Dialogues in Clinical Neuroscience, 17(3), 327-335. https://doi.org/10.31887 /DCNS.2015.17.3/bbandelow

Banerjee, S., \& Argáez, C. (2017). Neurofeedback and biofeedback for mood and anxiety disorders: A review of clinical effectiveness and guidelines. Ottawa, ON: Canadian Agency for Drugs and Technologies in Health.

Begemann, M. J. H., Florisse, E. J. R., van Lutterveld, R., Kooyman, M., \& Sommer, I. E. (2016). Efficacy of EEG neurofeedback in psychiatry: A comprehensive overview and meta-analysis. Translational Brain Rhythmicity, 1(1), 19-29. https://doi.org/10.15761/TBR.1000105

Bell, A. N., Moss, D., \& Kallmeyer, R. J. (2019). Healing the neurophysiological roots of trauma: A controlled study examining LORETA $z$-score neurofeedback and HRV biofeedback for chronic PTSD. NeuroRegulation, 6(2), 54-70. https://doi.org/10.15540/nr.6.2.54

Buchan, H., Sunderland, M., Carragher, N., Batterham, P., \& Slade, T. (2014). Investigating age-related differences in responses to screening items for internalising disorders in three national surveys. Journal of Affective Disorders, 152154, 229-236. https://doi.org/10.1016/j.jad.2013.09.015

Buzsáki, G., \& Watson, B. O. (2012). Brain rhythms and neural syntax: Implications for efficient coding of cognitive content and neuropsychiatric disease. Dialogues in Clinical Neuroscience, 14(4), 345-367. https://doi.org/10.31887 /DCNS.2012.14.4/gbuzsaki

Carragher, N., Krueger, R. F., Eaton, N. R., \& Slade, T. (2015). Disorders without borders: Current and future directions in the meta-structure of mental disorders. Social Psychiatry and Psychiatric Epidemiology, 50(3), 339-350. https://doi.org /10.1007/s00127-014-1004-z

Cheon, E. J., Choi, J. H., Lee, G. W., Koo, B. H., Seo, W. S., Kim, H. G., ... Keum, M. S. (2017). P.2.f.009 - Neurofeedback treatment on depressive symptoms and functional recovery and brain-derived neurotrophic factor in treatment-resistant major depression. European Neuropsychopharmacology, 27(Suppl. 4), S851. https://doi.org/10.1016/S0924977X(17)31529-8

Chiba, T., Kanazawa, T., Koizumi, A., Ide, K., TaschereauDumouchel, V., Boku, S., ... Kawato, M. (2019). Current status of neurofeedback for post-traumatic stress disorder: A systematic review and the possibility of decoded neurofeedback. Frontiers in Human Neuroscience, 13, 233. https://doi.org/10.3389/fnhum.2019.00233

Collura, T. F. (2013). Technical foundations of neurofeedback. London, UK: Taylor \& Francis.

Cosgrove, V. E., Rhee, S. H., Gelhorn, H. L., Boeldt, D., Corley, R. C., Ehringer, M. A., ... Hewitt, J. K. (2011). Structure and etiology of co-occurring internalizing and externalizing disorders in adolescents. Journal of Abnormal Child Psychology, 39(1), 109-123. https://doi.org/10.1007/s10802010-9444-8

Demyttenaere, K., Bruffaerts, R., Posada-Villa, J., Gasquet, I., Kovess, V., Lepine, J. P., ... Chatterji, S. (2004). Prevalence, severity, and unmet need for treatment of mental disorders in the World Health Organization World Mental Health Surveys. JAMA, 291(21), 2581-2590. https://doi.org/10.1001 /jama.291.21.2581

Duffy, M. E., Twenge, J. M., \& Joiner, T. E. (2019). Trends in mood and anxiety symptoms and suicide-related outcomes among U.S. undergraduates, 2007-2018: Evidence from two national surveys. Journal of Adolescent Health, 65(5), 590598. https://doi.org/10.1016/j.jadohealth.2019.04.033

Enriquez-Geppert, S., Huster, R. J., \& Herrmann, C. S. (2017). EEG-neurofeedback as a tool to modulate cognition and behavior: A review tutorial. Frontiers in Human Neuroscience, 11, 51. https://doi.org/10.3389/fnhum.2017.00051

Ghaziri, J., \& Thibault, R. T. (2019). 19 - Neurofeedback: An inside perspective. In A. Raz \& R. T. Thibault (Eds.), Casting light on the dark side of brain imaging (pp. 113-116): Cambridge, MA: Academic Press. https://doi.org/10.1016 /B978-0-12-816179-1.00019-0

Haidt, J., \& Allen, N. (2020). Scrutinizing the effects of digital technology on mental health. Nature, 578, 226-227. https://doi.org/10.1038/d41586-020-00296-X

Haller, S. P. W., Cohen Kadosh, K., Scerif, G., \& Lau, J. Y. F. (2015). Social anxiety disorder in adolescence: How developmental cognitive neuroscience findings may shape understanding and interventions for psychopathology. Developmental Cognitive Neuroscience, 13(C), 11-20. https://doi.org/10.1016/j.dcn.2015.02.002

Higgins, J. P. T., Thomas, J., Chandler, J., Cumpston, M., Li, T., Page, M. J., \& Welch, V. A. (Eds.). (2020). Cochrane handbook for systematic reviews of interventions (version 6.1). Available from www.training.cochrane.org/handbook

Holtmann, M., Sonuga-Barke, E., Cortese, S., \& Brandeis, D. (2014). Neurofeedback for ADHD: A review of current Evidence. Child and Adolescent Psychiatric Clinics of North America, 23(4), 789-806. https://doi.org/10.1016 /j.chc.2014.05.006

Hou, Y., Zhang, S., Li, N., Huang, Z., Wang, L., \& Wang, Y. (2021). Neurofeedback training improves anxiety trait and depressive symptom in GAD. Brain and Behavior, e02024. https://doi.org/10.1002/brb3.2024

Insel, T. R., \& Wang, P. S. (2010). Rethinking mental illness. JAMA, 303(19), 1970-1971. https://doi.org/10.1001 /jama.2010.555

losifescu, D. V. (2011). Electroencephalography-derived biomarkers of antidepressant response. Harvard Review of Psychiatry, 19(3), 144-154. https://doi.org/10.3109 /10673229.2011.586549

James, A. C., James, G., Cowdrey, F. A., Soler, A., \& Choke, A. (2015). Cognitive behavioural therapy for anxiety disorders in children and adolescents. Cochrane Database of Systematic Reviews, 2015(2), CD004690. https://doi.org/10.1002 /14651858.CD004690.pub4

Jokić-Begić, N., \& Begić, D. (2003). Quantitative electroencephalogram ( $\mathrm{qEEG}$ ) in combat veterans with posttraumatic stress disorder (PTSD). Nordic Journal of Psychiatry, 57(5), 351-355. https://doi.org/10.1080 108039480310002688

Kerson, C., \& Collaborative Neurofeedback Group. (2013). A proposed multisite double-blind randomized clinical trial of neurofeedback for ADHD: Need, rationale, and strategy. Journal of Attention Disorders, 17(5), 420-436. https://doi.org/10.1177/1087054713482580

Kessler, R. C., Aguilar-Gaxiola, S., Alonso, J., Chatterji, S., Lee, S., Ormel, J., ... Wang, P. S. (2009). The global burden of mental disorders: An update from the WHO World Mental Health (WMH) Surveys. Epidemiologia e psichiatria sociale (Epidemiology and Psychiatric Sciences), 18(1), 23-33. https://doi.org/10.1017/s1121189x00001421

Kessler, R. C., Angermeyer, M., Anthony, J. C., De Graaf, R., Demyttenaere, K., Gasquet, I., ... Ustün, T. B. (2007). Lifetime 
prevalence and age-of-onset distributions of mental disorders in the World Health Organization's World Mental Health Survey Initiative. World Psychiatry, 6(3), 168-176.

Kessler, R. C., Berglund, P., Demler, O., Jin, R., Merikangas, K. R., \& Walters, E. E. (2005). Lifetime prevalence and age-ofonset distributions of DSM-IV disorders in the National Comorbidity Survey Replication. Archives of General Psychiatry, 62(6), 593-602. https://doi.org/10.1001 /archpsyc.62.6.593

Keyes, K. M., Gary, D., O'Malley, P. M., Hamilton, A., \& Schulenberg, J. (2019). Recent increases in depressive symptoms among US adolescents: Trends from 1991 to 2018. Social Psychiatry and Psychiatric Epidemiology, 54(8), 987-996. https://doi.org/10.1007/s00127-019-01697-8

Kotov, R., Krueger, R. F., Watson, D., Achenbach, T. M., Althoff, R. R., Bagby, R. M., ... Zimmerman, M. (2017). The Hierarchical Taxonomy of Psychopathology (HiTOP): A dimensional alternative to traditional nosologies. Journal of Abnormal Psychology, 126(4), 454-477. https://doi.org /10.1037/abn0000258

Kris, N. (2018, December 4). 'Once in a generation': The crucial passages from the mental health inquiry. https://thespinoff.co.nz/politics/04-12-2018/once-in-ageneration-the-crucial-passages-from-the-mental-healthinquiry/

Krueger, R. F., \& Eaton, N. R. (2015). Transdiagnostic factors of mental disorders. World Psychiatry, 14(1), 27-29. https://doi.org/10.1002/wps.20175

Lancet Global Mental Health Group, Chisholm, D., Flisher, A. J., Lund, C., Patel, V., Saxena, S., ... Tomlinson, M. (2007). Scale up services for mental disorders: A call for action. The Lancet, 370(9594), 1241-1252. https://doi.org/10.1016 /S0140-6736(07)61242-2

Marzbani, H., Marateb, H. R., \& Mansourian, M. (2016). Neurofeedback: A comprehensive review on system design, methodology and clinical applications. Basic and Clinical Neuroscience, 7(2), 143-158. https://doi.org/10.15412 /J.BCN.03070208

Möller, H.-J., Bandelow, B., Volz, H.-P., Barnikol, U. B., Seifritz, E., \& Kasper, S. (2016). The relevance of 'mixed anxiety and depression' as a diagnostic category in clinical practice. European Archives of Psychiatry and Clinical Neuroscience, 266(8), 725-736. https://doi.org/10.1007/s00406-016-0684-7

Neurofeedback Collaborative Group, Arnold, L. E., Arns, M., Barterian, J., Bergman, R., Black, S., ... Williams, C. E. (2020). Double-blind placebo-controlled randomized clinical trial of neurofeedback for attention-deficit/hyperactivity disorder with 13-month follow-up. Journal of the American Academy of Child \& Adolescent Psychiatry. https://doi.org/10.1016/j.jaac.2020.07.906

Noohi, S., Miraghaie, A. M., Arabi, A., \& Nooripour, R. (2017). Effectiveness of neuro-feedback treatment with alpha/theta method on PTSD symptoms and their executing function. Biomedical Research (India), 28(5), 2019-2027.

Omejc, N., Rojc, B., Battaglini, P. P., \& Marusic, U. (2019). Review of the therapeutic neurofeedback method using electroencephalography: EEG neurofeedback. Bosnian Journal of Basic Medical Sciences, 19(3), 213-220. https://doi.org/10.17305/bjbms.2018.3785

Orndorff-Plunkett, F., Singh, F., Aragón, O. R., \& Pineda, J. A. (2017). Assessing the effectiveness of neurofeedback training in the context of clinical and social neuroscience. Brain Sciences, 7(8), 95. https://doi.org/10.3390/brainsci7080095

Panisch, L. S., \& Hai, A. H. (2018). The effectiveness of using neurofeedback in the treatment of post-traumatic stress disorder: A systematic review. Trauma, Violence, \& Abuse, 21(3), 541-550. https://doi.org/10.1177/1524838018781103

Peters, S. K., Dunlop, K., \& Downar, J. (2016). Cortico-striatalthalamic loop circuits of the salience network: A central pathway in psychiatric disease and treatment. Frontiers in
Systems Neuroscience, 10, 104. https://doi.org/10.3389 /fnsys.2016.00104

Pfeifer, J. H., \& Allen, N. B. (2020). Puberty initiates cascading relationships between neurodevelopmental, social, and internalizing processes across adolescence. Biological Psychiatry, $\quad$ 89(2), $\quad 99-108 . \quad$ https://doi.org/10.1016 /j.biopsych.2020.09.002

Pigott, H. E., Cannon, R., \& Trullinger, M. (2018). The fallacy of sham-controlled neurofeedback trials: A reply to Thibault and colleagues (2018). Journal of Attention Disorders, 25(3), 448457. https://doi.org/10.1177/1087054718790802

Pinter, A., Szatmari, S., Jr., Horvath, T., Penzlin, A. I., Barlinn, K., Siepmann, M., \& Siepmann, T. (2019). Cardiac dysautonomia in depression - Heart rate variability biofeedback as a potential add-on therapy. Neuropsychiatric Disease and Treatment, $15, \quad 1287-1310 . \quad$ https://doi.org/10.2147 /NDT.S200360

Pizzagalli, D. A., Nitschke, J. B., Oakes, T. R., Hendrick, A. M., Horras, K. A., Larson, C. L., ... Davidson, R. J. (2002). Brain electrical tomography in depression: The importance of symptom severity, anxiety, and melancholic features. Biological Psychiatry, 52(2), 73-85. https://doi.org/10.1016 IS0006-3223(02)01313-6

Reiter, K., Andersen, S. B., \& Carlsson, J. (2016). Neurofeedback treatment and posttraumatic stress disorder: Effectiveness of neurofeedback on posttraumatic stress disorder and the optimal choice of protocol. The Journal of Nervous and Mental Disease, 204(2), 69-77. https://doi.org/10.1097 /NMD.0000000000000418

Rhee, S. H., Lahey, B. B., \& Waldman, I. D. (2015). Comorbidity among dimensions of childhood psychopathology: Converging evidence from behavior genetics. Child Development Perspectives, 9(1), 26-31. https://doi.org /10.1111/cdep.12102

Ros, T., Frewen, P., Théberge, J., Michela, A., Kluetsch, R., Mueller, A., ... Lanius, R. A. (2017). Neurofeedback tunes scale-free dynamics in spontaneous brain activity. Cerebral Cortex, 27(10), 4911-4922. https://doi.org/10.1093/cercor /bhw285

Schoenberg, P. L. A., \& David, A. S. (2014). Biofeedback for psychiatric disorders: A systematic review. Applied Psychophysiology and Biofeedback, 39(2), 109-135. https://doi.org/10.1007/s10484-014-9246-9

Schönenberg, M., Wiedemann, E., Schneidt, A., Scheeff, J., Logemann, A., Keune, P. M., \& Hautzinger, M. (2017a). Confusion regarding operant conditioning of the EEG Authors' reply. The Lancet Psychiatry, 4(12), 897-898). https://doi.org/10.1016/S2215-0366(17)30437-6

Schönenberg, M., Wiedemann, E., Schneidt, A., Scheeff, J., Logemann, A., Keune, P. M., \& Hautzinger, M. (2017b). Neurofeedback, sham neurofeedback, and cognitivebehavioural group therapy in adults with attention-deficit hyperactivity disorder: A triple-blind, randomised, controlled trial. The Lancet Psychiatry, 4(9), 673-684. https://doi.org /10.1016/S2215-0366(17)30291-2

Sherlin, L. H., Arns, M., Lubar, J., Heinrich, H., Kerson, C., Strehl, U., \& Sterman, M. B. (2011). Neurofeedback and basic learning theory: Implications for research and practice. Journal of Neurotherapy, 15(4), 292-304. https://doi.org $/ 10.1080 / 10874208.2011 .623089$

Sitaram, R., Ros, T., Stoeckel, L., Haller, S., Scharnowski, F., Lewis-Peacock, J., ... Sulzer, J. (2016). Closed-loop brain training: The science of neurofeedback. Nature Reviews Neuroscience, 18, 86-100. https://doi.org/10.1038 /nrn.2016.164

Szewczyk, R. Ł., Ratomska, M., \& Jaśkiewicz, M. (2018). The neglected problem of the neurofeedback learning (in)ability. In W. Hunek \& S. Paskiel (Eds.), Biomedical Engineering and Neuroscience, Advances in Intelligent Systems and 
Computing (vol. 720, pp. 45-58). Switzerland: Springer, Cham. https://doi.org/10.1007/978-3-319-75025-5_6

Thibault, R. T., Lifshitz, M., \& Raz, A. (2016). The self-regulating brain and neurofeedback: Experimental science and clinical promise. Cortex, 74, 247-261. https://doi.org/10.1016 /j.cortex.2015.10.024

Thibault, R. T., Veissière, S., Olson, J. A., \& Raz, A. (2018). Treating ADHD with suggestion: Neurofeedback and placebo therapeutics. Journal of Attention Disorders, 22(8), 707-711. https://doi.org/10.1177/1087054718770012

Tiller, J. W. G. (2013). Depression and anxiety. The Medical Journal of Australia, 199(S6), S28-S31. https://doi.org $/ 10.5694 / \mathrm{mja} 12.10628$

Tolin, D. F., Davies, C. D., Moskow, D. M., \& Hofmann, S. G. (2020). Biofeedback and neurofeedback for anxiety disorders: A quantitative and qualitative systematic review. In Y.-K. Kim (Ed.), Anxiety disorders: Rethinking and understanding recent discoveries (pp. 265-289). Singapore: Springer Singapore. https://doi.org/10.1007/978-981-32-9705-0_16

Trullinger, M., Novian, A., Russell-Chapin, L., \& Pradhan, D. (2019). Perspectives on Type III statistical errors: Exaggerating the effects of placebo in neurofeedback. NeuroRegulation, 6(1), 38-41. https://doi.org/10.15540 /nr.6.1.38

Twenge, J. M., Cooper, A. B., Joiner, T. E., Duffy, M. E., \& Binau, S. G. (2019). Age, period, and cohort trends in mood disorder indicators and suicide-related outcomes in a nationally representative dataset, 2005-2017. Journal of Abnormal Psychology, 128(3), 185-199. https://doi.org/10.1037 labn0000410

van der Kolk, B. A., Hodgdon, H., Gapen, M., Musicaro, R., Suvak, M. K., Hamlin, E., \& Spinazzola, J. (2016). A randomized controlled study of neurofeedback for chronic PTSD. PLOS ONE, 11(12), e0166752. https://doi.org/10.1371 /journal.pone.0166752

Van Doren, J., Arns, M., Heinrich, H., Vollebregt, M. A., Strehl, U., \& K. Loo, S. (2019). Sustained effects of neurofeedback in ADHD: A systematic review and meta-analysis. European
Child \& Adolescent Psychiatry, 28(3), 293-305. https://doi.org /10.1007/s00787-018-1121-4

Wahbeh, H., \& Oken, B. S. (2013). Peak high-frequency HRV and peak alpha frequency higher in PTSD. Applied Psychophysiology and Biofeedback, 38(1), 57-69. https://doi.org/10.1007/s10484-012-9208-z

Wang, S.-Y., Lin, I.-M., Fan, S.-Y., Tsai, Y.-C., Yen, C.-F., Yeh, Y.-C., ... Lin, H.-C. (2019). The effects of alpha asymmetry and high-beta down-training neurofeedback for patients with the major depressive disorder and anxiety symptoms. Journal of Affective Disorders, 257, 287-296. https://doi.org/10.1016 /j.jad.2019.07.026

Wells, J. E., Oakley Browne, M. A., Scott, K. M., McGee, M. A., Baxter, J., \& Kokaua, J. (2006). Te Rau Hinengaro: The New Zealand Mental Health Survey: Overview of methods and findings. Australian \& New Zealand Journal of Psychiatry, 40(10), $\quad 835-844 . \quad$ https://doi.org/10.1080/j.14401614.2006.01902.x

Witte, M., Kober, S. E., \& Wood, G. (2018). Noisy but not placebo: Defining metrics for effects of neurofeedback. Brain, 141(5), e40. https://doi.org/10.1093/brain/awy060

World Health Organization. (2018). ICD-11. International classification of diseases for mortality and morbidity statistics (11th Revision). Retrieved from https://icd.who.int/browse11/l$\mathrm{m} / \mathrm{en}$

Zuberer, A., Brandeis, D., \& Drechsler, R. (2015). Are treatment effects of neurofeedback training in children with ADHD related to the successful regulation of brain activity? A review on the learning of regulation of brain activity and a contribution to the discussion on specificity. Frontiers in Human Neuroscience, 9, 135 . https://doi.org/10.3389 /fnhum.2015.00135

Received: February 25, 2021

Accepted: March 18, 2021

Published: March 29, 2021 